\title{
Effect of laparoscopic myomectomy on serum levels of IL-6 and TAC, and ovarian function
}

\author{
YANLING HU*, LIYAO YU*, FEN XIA, FENGQI LIANG, CHAO CHENG, YUHUA HUANG and LINQI XIAO \\ Department of Gynaecology and Obstetrics, The Central Hospital of Wuhan, Tongji Medical College, \\ Huazhong University of Science and Technology, Wuhan, Hubei 430030, P.R. China
}

Received January 2, 2019; Accepted July 18, 2019

DOI: $10.3892 /$ etm.2019.7941

\begin{abstract}
Effect of laparoscopic myomectomy on the serum levels of interleukin-6 (IL-6) and total antioxidant capacity (TAC), and the ovarian function of patients with uterine fibroids was studied. Ninety patients with uterine fibroids admitted to The Central Hospital of Wuhan, Tongji Medical College, Huazhong University of Science and Technology, from March 2013 to July 2014, were randomly divided into the experimental group $(n=45)$ and the control group $(n=45)$. The experimental group was treated with laparoscopic myomectomy, and the control group was treated with abdominal myomectomy. ELISA was used for detecting IL-6 and TAC content. Radioimmunoassay (RIA) was used for detecting serum ovarian function indicators, including estradiol $\left(\mathrm{E}_{2}\right)$, follicle-stimulating hormone (FSH) and luteinizing hormone ( $\mathrm{LH})$. Patients in the experimental group had significantly lower intraoperative blood loss, postoperative exhaust time, number of days to recovery and return to work, number of intraoperative fibroids removed, and length of stay than those in the control group $(\mathrm{P}<0.05)$. TAC expression level was significantly higher in the experimental group than that in the control group, on the 1st, 3rd and 5th day after surgery $(\mathrm{P}<0.05)$, while IL-6 expression was significantly lower in the experimental group than that in the control group at the same time points $(\mathrm{P}<0.05)$. FSH and $\mathrm{LH}$ expression levels were significantly lower in the experimental group than those in the control group $(\mathrm{P}<0.05)$ at 1,3 and 6 months after surgery, while $\mathrm{E}_{2}$ expression level was significantly higher in the experimental group than that in the control group at the same time points $(\mathrm{P}<0.05)$. The incidence of postoperative complications of patients in the experimental group was significantly lower than that in the control group $(\mathrm{P}<0.05)$. Patients in the
\end{abstract}

Correspondence to: Dr Linqi Xiao, Department of Gynaecology and Obstetrics, The Central Hospital of Wuhan, Tongji Medical College, Huazhong University of Science and Technology, 26 Shengli Street, Wuhan, Hubei 430030, P.R. China

E-mail: eqa5jq@163.com

*Contributed equally

Key words: myomectomy, interleukin-6, ovarian function, octagonal cyclophosphamide, uterine fibroid experimental group had significantly higher successful pregnancy rate than those in the control group $\left(\chi^{2}=6.75, \mathrm{P}<0.05\right)$. Reducing its effect on ovarian function, serum pain index and oxidative damage index of patients with uterine fibroid, laparoscopic myomectomy also reduces the incidence of postoperative complications and increases the pregnancy rate.

\section{Introduction}

Uterine fibroid, also known as fibromyoma, is a benign tumor commonly found in female patients (1). According to a study, the incidence of uterine fibroids reaches 233-556/10,000 (2). It has been reported that $5.4-77 \%$ of females have uterine fibroids (3). Uterine fibroid is a benign tumor of gynecology, however, its pathogenesis remains unclear. In clinical practice, uterine fibroids can be divided into single and multiple uterine fibroids. Multiple uterine fibroids are more common, often with irregular menstruation, urination disorder and dysporia, abortion and infertility (4). A study (5) has found that uterine fibroids are more common among females with childbearing age, widowhood and uncoordinated sexual life. The occurrence and development of uterine fibroids are caused by the interaction of a number of factors. As the incidence of uterine fibroids increases, the therapeutic methods and clinical efficacy have become a research hotspot. Traditional laparotomy, which is not in line with the minimally invasive concept, has gradually been replaced by arteriosus minimally invasive surgery (6).

The rapid development of laparoscopic surgery has resulted in less damage to the pelvic tissues and organs, avoiding complications caused by the aggravation of postoperative stress injury $(7,8)$. The postoperative effect of laparoscopic myomectomy is similar to the clinical effect of conventional laparotomy. Research has shown that it has the same rejection effect on fibroids $(9,10)$. However, there is still a lack of relevant studies on serological inflammatory factors or long-term clinical pregnancy outcomes after laparoscopic surgery has been performed on patients with uterine fibroids. Interleukin-6 (IL-6) is an important indicator reflecting body pain. When patients have pain/stress responses, IL- 6 is to a certain degree abnormally increased. The expression level of IL- 6 in the body is positively correlated with pain (11). Total antioxidant capacity (TAC) is an important evaluation index that reflects the oxidative damage in the body (12). Moreover, the influence degree of the surgery on the surrounding tissues reflects the ovarian function, and 
monitoring the ovarian function and the oxidative damage of patients can reflect the safety of the surgery (13). In order to further reveal the clinical effect of laparoscopic myomectomy, 90 patients with uterine fibroid treated in The Central Hospital of Wuhan (Wuhan, China), from March 2013 to July 2014, were enrolled to investigate the effect of laparoscopic myomectomy on serum levels of IL-6 and TAC, and the ovarian function.

\section{Patients and methods}

General information. Ninety patients with uterine fibroids admitted to the Central Hospital of Wuhan, Tongji Medical College, Huazhong University of Science and Technology, from March 2013 to July 2014, were enrolled in the present study. Patients were randomly divided into the experimental group $(n=45)$ and the control group $(n=45)$. The patients in the experimental group were treated with laparoscopic myomectomy. In the experimental group, the average age of the patients was $38.3 \pm 4.12$ years, the course of disease was $4.69 \pm 1.76$ years, the number of fibroids was $4.57 \pm 1.34$ and the average fibroid diameter was $4.67 \pm 1.98 \mathrm{~cm}$. There were 29 patients with single uterine fibroid and 16 patients with multiple uterine fibroids. Pathological types: 14 patients with intramural fibroid, 17 patients with subserosal fibroid, 9 patients with broad ligament myoma and 5 patients with submucosal myoma. The patients in the control group received abdominal myomectomy. In the control group, the average age of the patients was $39.4 \pm 4.54$ years, the course of disease was $4.58 \pm 1.83$ years, the number of fibroids was $4.59 \pm 1.31$ and the average fibroid diameter was $4.72 \pm 1.87 \mathrm{~cm}$. There were 31 patients with single uterine fibroid and 14 patients with multiple uterine fibroids. Pathological types: 13 patients with intramural fibroid, 16 patients with subserosal fibroid, 10 patients with broad ligament myoma and 6 patients with submucosal myoma.

Inclusion criteria: Patients diagnosed with uterine fibroids by B-ultrasound or hysteroscopy; patients who had not undergone pelvic surgery; patients who had fertility requirements; patients with increased menstruation, irregular bleeding or lower abdominal mass; patients with surgical tolerance; patients not treated with hormonal drugs before surgery; patients with coagulation dysfunction or systemic blood diseases; patients with special fibroid locations.

Exclusion criteria: Patients with other malignant tumors; patients with heart, liver, kidney or other important organs with severe functional insufficiency; patients with mental disorder who were unable to communicate; pregnant or lactating women; patients with ovarian cyst, endometriosis and coagulation dysfunction.

The study was approved by the Ethics Committee of the Central Hospital of Wuhan, Tongji Medical College, Huazhong University of Science and Technology. Patients who participated in this study had complete clinical data. Signed informed consents were obtained from the patients or their guardians.

Treatments. Patients in the experimental group were treated with laparoscopic myomectomy. Each patient was subjected to general anesthesia, and placed in a stone bladder position. The head was kept low and the feet high, with a tilt angle of $15^{\circ}-30^{\circ}$. Shoulder pads were used to fix the shoulders of the patient if necessary. Then, with the patient's navel mouth, left and right McBurney's points as the operating path, a 2-cm incision was made at the lower edge of the umbilical hole. A pneumoperitoneum needle was punctured to establish artificial pneumoperitoneum, and the pressure was controlled at 14-15 mmHg. Laparoscope was placed from the umbilical portion of the patient, and an incision was respectively made in the avascular region of the patient's anti-McBurney's point and at the McBurney's point, with instruments placed. During surgery, the seromuscular layer of the fibroid was unilaterally incised to the tumor nucleus. The fibroid was fixed by grasping forceps and peeled off. The bleeding of the wound was stopped by electrocoagulation and the wound was sutured layer-by-layer, with fibroids cut by a rotary cutter from the abdominal incision. Repeated check was carried out to confirm whether fibroids were taken cleanly. The abdominal cavity was rinsed after confirming the removal. Active bleeding in the patient was checked. After deflation, the laparoscope and instruments were removed, and the puncture wound was sutured. Antibiotics were administered after surgery to prevent infection of the wound, and oxytocin to promote uterine contraction.

Patients in the control group were treated with abdominal myomectomy. Each patient was subjected to combined spinal and epidural anesthesia, and placed in a supine position. After disinfection, a 5-8 cm incision was made in the lower abdomen of the patient. Tissues were cut layer-by-layer to expose the surgical field of fibroids. The number, size and location of fibroids were carefully checked. A fibroid turn was used to remove fibroids, and electrocoagulation to stop bleeding. The abdominal cavity was rinsed after confirming the removal. Active bleeding in the patient was checked. A bio-absorbed thread (Johnson \& Johnson) was used to suture the uterine wound. In order to prevent tissue adhesion, 'Rui Shu Kang' ointment (Hebei Ruinuo Medical Instrument Co., Ltd.) was applied to the wound. The abdominal cavity was closed layer-by-layer. Antibiotics were administered after surgery to prevent infection of the wound, and oxytocin to promote uterine contraction.

Detection methods. Elbow venous blood $(5 \mathrm{ml})$ was extracted from the patients on an empty stomach before surgery, and on the 1st, 3rd and 5th day after surgery. The samples were centrifuged at $1,006.2 \mathrm{x} \mathrm{g}$ at $4^{\circ} \mathrm{C}$ for $10 \mathrm{~min}$ and the supernatant was collected. ELISA was used for the detection of the serum levels of IL-6 and TAC. Detection kits (SBJ-H0465 and SBJ-H129) were purchased from Nanjing Senbeijia Biological Co., Ltd. The specific detection steps followed were in strict accordance with the manufacturer's instructions. Elbow venous blood $(5 \mathrm{ml})$ was also extracted from the patients on an empty stomach before surgery, and on the 1st, 3rd and 6th month after surgery. The samples were centrifuged at $1,006.2 \mathrm{x}$ at $4^{\circ} \mathrm{C}$ for $10 \mathrm{~min}$ to collect the supernatant. Radioimmunoassay (RIA) was used for the detection of the serum ovarian function indicators, including luteinizing hormone ( $\mathrm{LH})$, follicle-stimulating hormone (FSH) and estradiol $\left(\mathrm{E}_{2}\right)$.

Follow-up and outcome measures. Patients were followed up via hospital review and telephone. Patients with recurrence were promptly treated, and examinations were carried out to complete the relevant prognostic data. The follow-up ended on August 1, 2017. The pregnancy outcomes of patients in the two groups were recorded for 3 years. The expression of serum IL-6 
Table I. Comparison of the general clinical data of patients between the two groups [mean $\pm \mathrm{SD}, \mathrm{n}(\%)$ ].

\begin{tabular}{|c|c|c|c|c|}
\hline Characteristics & $\begin{array}{l}\text { Experimental group } \\
\qquad(\mathrm{n}=45)\end{array}$ & $\begin{array}{l}\text { Control group } \\
(\mathrm{n}=45)\end{array}$ & $\chi^{2} / \mathrm{t}$ value & P-value \\
\hline Age (years) & $38.3 \pm 4.12$ & $39.4 \pm 4.54$ & -0.345 & 0.842 \\
\hline Type of hukou & & & 0.443 & 0.506 \\
\hline Local hukou & $31(68.89)$ & $28(62.22)$ & & \\
\hline Non-local hukou & $14(31.11)$ & $17(37.78)$ & & \\
\hline Course of disease (years) & $4.69 \pm 1.76$ & $4.58 \pm 1.83$ & 0.872 & 0.130 \\
\hline No. of fibroids & $4.57 \pm 1.34$ & $4.59 \pm 1.31$ & -0.498 & 0.574 \\
\hline Fibroid diameter $(\mathrm{cm})$ & $4.67 \pm 1.98$ & $4.72 \pm 1.87$ & -1.468 & 0.266 \\
\hline \multicolumn{5}{|l|}{ Fibroid type } \\
\hline Intramural fibroid & $14(31.11)$ & $13(28.89)$ & 0.053 & 0.818 \\
\hline Subserosal fibroid & $17(37.78)$ & $16(35.56)$ & 0.048 & 0.827 \\
\hline Broad ligament myoma & $9(20.00)$ & $10(22.22)$ & 0.067 & 0.796 \\
\hline Submucosal myoma & $5(11.11)$ & $6(13.33)$ & 0.104 & 0.748 \\
\hline Type & & & 0.200 & 0.655 \\
\hline Single & $29(64.44)$ & $31(68.89)$ & & \\
\hline Multiple & $16(35.56)$ & $14(31.11)$ & & \\
\hline \multicolumn{5}{|l|}{ Fibroid location } \\
\hline Posterior wall & $20(44.44)$ & $21(46.67)$ & 0.045 & 0.832 \\
\hline Anterior wall & $18(40.00)$ & $15(33.33)$ & 0.431 & 0.512 \\
\hline Fundus & $7(15.56)$ & $9(20.00)$ & 0.304 & 0.581 \\
\hline
\end{tabular}

and TAC, and the changes in ovarian function indicators $\mathrm{E}_{2}$, FSH and LH of the patients in the two groups were observed. Postoperative complications of patients were recorded in detail.

Statistical analysis. SPSS 17.0 statistical software (IBM Corp.) was used for the analysis of the experimental data. Enumeration data were expressed as n (\%), and Chi-square test was used for comparisons between two groups. Measurement data were expressed as mean $\pm \mathrm{SD}$, and t-test was used to analyze data between two groups. One-way ANOVA and Dunnett's post hoc test were used for comparisons among multiple time points. The mean values before surgery were used as the control for the comparisons of the data of the different time points within the group. $\mathrm{P}<0.05$ was considered to indicate a statistically significant difference.

\section{Results}

Comparison of the general clinical data of patients between the two groups. There were no statistically significant differences between the experimental and control group in terms of age, type of registered permanent residence, course of disease, number of fibroids, fibroid location, diameter and type $(\mathrm{P}>0.05)$. Thus, the patients in the two groups were comparable (Table I).

Comparison of the surgical conditions of patients between the two groups. There were no statistically significant differences between the experimental and control group in terms of surgery time, postoperative leaving-bed time, and self-care time $(\mathrm{P}>0.05)$. As can be seen from Table II, the intraoperative blood loss, postoperative exhaust time, number of days to recovery and return to work, number of intraoperative fibroids removed, and length of stay of patients in the experimental group were $109.24 \pm 18.76 \mathrm{ml}, 18.45 \pm 3.12 \mathrm{~h}, 27.45 \pm 8.31$ days, $6.13 \pm 1.42$ fibroids and $4.14 \pm 1.23$ days, respectively, and were significantly lower $(\mathrm{P}<0.05)$ than those in the control group, i.e., $148.24 \pm 32.16 \mathrm{ml}, 30.12 \pm 5.23 \mathrm{~h}, 40.01 \pm 9.79$ days, $8.16 \pm 1.31$ fibroids and $7.87 \pm 1.97$ days, respectively.

Comparison of the serum TAC and IL-6 expression levels of patients between the two groups. There was no statistically significant difference in the expression levels of TAC and IL-6 in the serum of patients between the experimental and control group before surgery $(\mathrm{P}>0.05)$ (Table III). Patients in the two groups had significantly lower TAC expression levels on the 1 st, 3rd and 5th day after surgery than that before surgery $(\mathrm{P}<0.05)$, and TAC expression levels were significantly higher in the experimental group than those in the control group on the 1st, 3rd and 5th day after surgery $(\mathrm{P}<0.05)$. Patients in the two groups had significantly higher IL-6 expression levels on the 1st, 3rd and 5th day after surgery than that before surgery $(\mathrm{P}<0.05)$, and IL-6 expression levels were significantly lower in the experimental group than those in the control group on the 1 st, 3rd and 5th day after surgery $(\mathrm{P}<0.05)$ (Table III).

Comparison of the ovarian function of patients between the two groups. Before surgery, there were no statistically significant differences in the expression levels of $\mathrm{FSH}, \mathrm{E}_{2}$ and LH of the patients between the experimental and control group ( $\mathrm{P}>0.05)$ (Table IV). Patients in the two groups had significantly higher FSH and LH expression levels on the 1st, 3rd and 6th month after surgery than those before surgery 
Table II. Comparison of the surgical conditions of patients between the two groups (mean \pm SD).

\begin{tabular}{|c|c|c|c|c|}
\hline Surgical conditions & $\begin{array}{l}\text { Experimental group } \\
\qquad(\mathrm{n}=45)\end{array}$ & $\begin{array}{l}\text { Control group } \\
\qquad(\mathrm{n}=45)\end{array}$ & t value & P-value \\
\hline Surgery time (min) & $75.67 \pm 13.36$ & $63.12 \pm 16.23$ & 5.129 & 0.272 \\
\hline Intraoperative blood loss (ml) & $109.24 \pm 18.76$ & $148.24 \pm 32.16$ & -6.159 & $0.003^{\mathrm{a}}$ \\
\hline Postoperative leaving-bed time (h) & $12.35 \pm 2.41$ & $13.56 \pm 3.04$ & -2.435 & 0.066 \\
\hline Self-care time (days) & $7.15 \pm 0.66$ & $6.84 \pm 0.76$ & 2.049 & 0.166 \\
\hline Postoperative exhaust time (h) & $18.45 \pm 3.12$ & $30.12 \pm 5.23$ & -17.527 & $0.001^{\mathrm{a}}$ \\
\hline No. of days to recovery and return to work & $27.45 \pm 8.31$ & $40.01 \pm 9.79$ & -8.682 & $0.027^{\mathrm{a}}$ \\
\hline No. of intraoperative fibroids removed & $6.13 \pm 1.42$ & $8.16 \pm 1.31$ & -7.824 & $0.031^{\mathrm{a}}$ \\
\hline Length of stay (days) & $4.14 \pm 1.23$ & $7.87 \pm 1.97$ & -9.581 & $<0.001^{\mathrm{a}}$ \\
\hline
\end{tabular}

${ }^{\mathrm{a}} \mathrm{P}<0.05$, significant difference between the experimental and control group.

Table III. Comparison of the serum TAC and IL-6 expression levels of patients between the two groups (mean $\pm \mathrm{SD}$ ).

\begin{tabular}{lcc}
\hline Groups & TAC $(\mathrm{kU} / \mathrm{l})$ & IL-6 $(\mathrm{pg} / \mathrm{ml})$ \\
\hline Control group & & \\
Before surgery & $13.65 \pm 1.56$ & $5.43 \pm 0.65$ \\
1 day after surgery & $9.44 \pm 0.97^{\mathrm{a}}$ & $9.01 \pm 1.02^{\mathrm{a}}$ \\
3 days after surgery & $8.21 \pm 0.98^{\mathrm{a}}$ & $9.46 \pm 1.28^{\mathrm{a}}$ \\
5 days after surgery & $9.28 \pm 0.91^{\mathrm{a}}$ & $8.81 \pm 0.98^{\mathrm{a}}$ \\
F value & 200.7 & 152.0 \\
P-value & $<0.001$ & $<0.001$ \\
Experimental group & & \\
Before surgery & $13.61 \pm 1.49$ & $5.32 \pm 0.70$ \\
1 day after surgery & $11.82 \pm 1.49^{\mathrm{a}, \mathrm{b}}$ & $6.53 \pm 0.63^{\mathrm{a}, \mathrm{b}}$ \\
3 days after surgery & $11.21 \pm 1.20^{\mathrm{a}, \mathrm{b}}$ & $7.01 \pm 0.89^{\mathrm{a}, \mathrm{b}}$ \\
5 days after surgery & $11.42 \pm 1.36^{\mathrm{a}, \mathrm{b}}$ & $6.67 \pm 0.79^{\mathrm{a}, \mathrm{b}}$ \\
F value & 27.82 & 42.39 \\
P-value & $<0.001$ & $<0.001$ \\
\hline
\end{tabular}

${ }^{a} \mathrm{P}<0.05$, compared with before surgery in the same group; ${ }^{\mathrm{b}} \mathrm{P}<0.05$, compared with the same time point in the control group. TAC, total antioxidant capacity; IL-6, interleukin-6.

$(\mathrm{P}<0.05)$, and $\mathrm{SH}$ and $\mathrm{LH}$ expression levels were significantly lower in the experimental group than those in the control group $(\mathrm{P}<0.05)$. Patients in the two groups had significantly lower $\mathrm{E}_{2}$ expression levels on the 1st, 3rd and 6th month after surgery than that before surgery $(\mathrm{P}<0.05)$, and $\mathrm{E}_{2}$ expression levels were significantly higher in the experimental group than those in the control group, with a statistically significant difference $(\mathrm{P}<0.05)$ (Table IV).

Comparison of the incidence of postoperative complications of patients between the two groups (Table V). There was only 1 patient with abdominal pain and 3 patients with postoperative fever after surgery in the experimental group, with the incidence of postoperative complications being $8.89 \%$. There were 3 patients with abdominal pain, 1 patient with wound infection, 1 patient with urinary system infection, 2 patients with pelvic adhesion and 5 patients with postoperative fever after surgery in the control group, with the incidence of postoperative complications being $26.66 \%$, significantly higher than that in the experimental group $(\mathrm{P}<0.05)$.

Comparison of the postoperative successful pregnancy rate of patients between the two groups (Fig. 1). Three years after surgery, 22 patients had successful pregnancy in the experimental group, with a pregnancy rate of $48.89 \%$; 14 patients had successful pregnancy in the control group, with a pregnancy rate of $31.11 \%$. Patients in the experimental group had a significantly higher successful pregnancy rate than those in the control group, with a statistically significant difference $\left(\chi^{2}=6.75, P<0.05\right)$.

\section{Discussion}

Uterine fibroid is a common benign tumor of female genitalia. Change of the age of menarche and people's living habits have increased the incidence of uterine fibroids, and in addition, patients with uterine fibroids are increasingly becoming younger (14). Uterine fibroid may be caused by interactions among sex hormone changes, smooth muscle cell (SMC) mutations and local growth factors (15). Changes in the sensitivity of local receptors for uterine SMCs and sex hormone disorder usually cause uterine fibroids. The sustained proliferation of SMCs is related to the long-term activation of estrogen receptors, further resulting in uterine fibroids (16). The long-term occurrence of uterine fibroid causes red-like changes and peeling-like degeneration, which greatly increase the risk of malignant lesions in uterine fibroids (17). Currently, surgical treatment is the preferred treatment for uterine fibroids (18). Therefore, laparoscopic surgery is an effective means to treat uterine fibroid clinically. Compared with traditional laparotomy, laparoscopic surgery has a small traction range, less trauma and is a fine surgery $(19,20)$. At present, there are studies on the clinical effect of laparoscopic surgery for treating uterine fibroids. However, there is lack of studies on serum IL-6 and TAC changes, and ovarian function.

Serum markers are important for the evaluation of body health. IL-6 is an important inflammatory response factor (21). Derived from mononuclear macrophages and SMCs, IL-6 
Table IV. Comparison of the ovarian function of patients between the two groups (mean \pm SD).

\begin{tabular}{lccc}
\hline Groups & FSH $(\mathrm{U} / \mathrm{l})$ & $\mathrm{E}_{2}(\mathrm{pmol} / \mathrm{l})$ & $\mathrm{LH}(\mathrm{U} / \mathrm{l})$ \\
\hline Control group & & & \\
Before surgery & $17.45 \pm 1.73$ & $291.24 \pm 13.23$ & $17.87 \pm 1.57$ \\
1 month after surgery & $23.78 \pm 2.02^{\mathrm{a}}$ & $225.12 \pm 12.46^{\mathrm{a}}$ & $24.77 \pm 2.08^{\mathrm{a}}$ \\
3 months after surgery & $26.37 \pm 2.19^{\mathrm{a}}$ & $221.12 \pm 12.32^{\mathrm{a}}$ & $25.98 \pm 2.12^{\mathrm{a}}$ \\
6 months after surgery & $23.83 \pm 1.94^{\mathrm{a}}$ & $230.07 \pm 12.52^{\mathrm{a}}$ & $24.82 \pm 1.81^{\mathrm{a}}$ \\
F value & 166.9 & 308.8 & 169.4 \\
P-value & $<0.001$ & $<0.001$ & $<0.001$ \\
Experimental group & & & $17.85 \pm 1.52$ \\
Before surgery & $17.42 \pm 1.69$ & $290.89 \pm 13.19$ & $20.31 \pm 1.55^{\mathrm{a}, \mathrm{b}}$ \\
1 month after surgery & $19.87 \pm 1.71^{\mathrm{a}, \mathrm{b}}$ & $258.98 \pm 11.21^{\mathrm{a}, \mathrm{b}}$ & $21.09 \pm 1.62^{\mathrm{a}, \mathrm{b}}$ \\
3 months after surgery & $21.31 \pm 1.77^{\mathrm{a}, \mathrm{b}}$ & $248.93 \pm 11.13^{\mathrm{a}, \mathrm{b}}$ & $20.86 \pm 1.98^{\mathrm{a}, \mathrm{b}}$ \\
6 months after surgery & $20.72 \pm 1.61^{\mathrm{a}, \mathrm{b}}$ & $272.69 \pm 11.43^{\mathrm{a}, \mathrm{b}}$ & 35.41 \\
F value & 45.85 & 107.3 & $<0.001$ \\
P-value & $<0.001$ & $<01$ & $<$ \\
\hline
\end{tabular}

${ }^{\mathrm{a}} \mathrm{P}<0.05$, compared with before surgery in the same group; ${ }^{\mathrm{b}} \mathrm{P}<0.05$, compared with the same time point in the control group. FSH, follicle-stimulating hormone; $\mathrm{E}_{2}$, estradiol; $\mathrm{LH}$, luteinizing hormone.

Table V. Comparison of the incidence of postoperative complications of patients between the two groups [n (\%)].

\begin{tabular}{|c|c|c|c|c|c|c|}
\hline Groups & $\begin{array}{l}\text { Abdominal } \\
\text { pain }\end{array}$ & $\begin{array}{l}\text { Wound } \\
\text { infection }\end{array}$ & $\begin{array}{l}\text { Urinary system } \\
\text { infection }\end{array}$ & $\begin{array}{c}\text { Pelvic } \\
\text { adhesion }\end{array}$ & $\begin{array}{c}\text { Postoperative } \\
\text { fever }\end{array}$ & Incidence \\
\hline Experimental group $(n=45)$ & $1(2.22)$ & $0(0.00)$ & $0(0.00)$ & $0(0.00)$ & $3(6.67)$ & $8.89 \%$ \\
\hline Control group $(n=45)$ & $3(6.67)$ & $1(2.22)$ & $1(2.22)$ & $2(4.44)$ & $5(11.11)$ & $26.66 \%$ \\
\hline$\chi^{2}$ value & & & & & & 10.98 \\
\hline P-value & & & & & & 0.001 \\
\hline
\end{tabular}

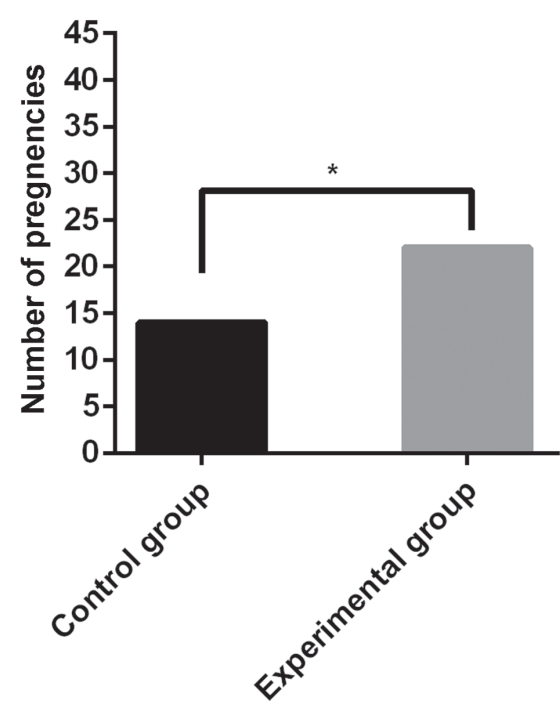

Figure 1. Comparison of the postoperative successful pregnancy rate of patients between the two groups. The pregnancy outcomes of patients in the two groups after surgery were recorded for 3 years. In total, 22 patients had successful pregnancy in the experimental group, with a pregnancy rate of $48.89 \%$, and 14 patients had successful pregnancy in the control group, with a pregnancy rate of $31.11 \%$. Patients in the experimental group had a significantly higher successful pregnancy rate than those in the control group, with a statistically significant difference $\left(\chi^{2}=6.75,{ }^{*} \mathrm{P}<0.05\right)$. stimulates B cells to produce antibodies, thereby activating and proliferating $\mathrm{T}$ cells, and stimulating the immune response of the body. Therefore, IL- 6 is a key factor in acute inflammatory responses (22). It has been reported that surgery causes certain trauma and an increase in IL-6 and other inflammatory factors, which induce inflammatory responses, affecting the recovery of the patients (23). Antioxidant defense substances in the body synergistically exert antioxidant effects, and the sum of their antioxidant capacity is called TAC. TAC, an important evaluation index for the oxidative damage of the body (24), consists of two major parts, enzymatic and non-enzymatic reactions (25), and is important for evaluating the efficacy of laparoscopic surgery. As an important reproductive organ, ovaries play a role in reproduction and endocrine effects. Its reproductive effects mainly include follicular recruitment, development, follicular maturation, ovulation, luteinization and degeneration. Its endocrine effects are mainly expressed in secreting estrogens, progesterone, androgens, peptide hormones and growth factors $(26,27)$. Therefore, it is particularly important to study the effect of myomectomy on the ovarian function.

In this study, the general clinical data of patients were analyzed and compared between the experimental and control group. There was no significant difference in the clinical data 
of patients between the two groups, which excluded basic interference for this study. The postoperative conditions of patients were compared between the experimental and control group. The results revealed that patients in the experimental group had significantly lower intraoperative blood loss, postoperative exhaust time, number of days to recovery and return to work, number of intraoperative fibroids removed, and length of stay compared with the control group. According to the study of Brucher et al (28), on the comparison of the clinical effect of laparoscopic myomectomy and abdominal myomectomy, the surgery time in the laparoscopic myomectomy group is longer than the conventional group; intraoperative blood loss, postoperative anal exhaust time and postoperative hospital stay are lower than the conventional group, and the total effective rate of the laparoscopic group is higher than that of the conventional group. Compared with abdominal myomectomy, laparoscopic surgery had smaller trauma and faster postoperative recovery of patients. There were no statistically significant differences in the expression levels of TAC and IL-6, and the ovarian function in the serum of patients between the two groups before surgery. Patients in the two groups had significantly lower TAC expression level after surgery than those before surgery, which was significantly higher in the experimental group than that in the control group. Patients in the two groups had significantly higher IL-6 expression level after surgery than that before surgery, which was significantly lower in the experimental group than that in the control group $(\mathrm{P}<0.05)$. In the study on the effect of laparoscopic myomectomy on ovarian function and trauma-related serum parameters, Dong (11) found that, serum pain-related indicators after surgery, including myocardial cell substance $\mathrm{P}(\mathrm{SP})$, IL-6 and prostaglandin $\mathrm{E}_{2}\left(\mathrm{PGE}_{2}\right)$ in laparoscopic myomectomy (observation group) were significantly lower than abdominal myomectomy (control group); serum oxidative damage index myoglobin (MYO) and serum ischemia modified albumin (IMA) at various time points after surgery in the observation group were lower than the control group, and TAC was higher than that in the control group. This is mainly because laparoscopic surgery has smaller trauma, less intraoperative blood loss and more complete hemostasis than traditional abdominal surgery. Therefore, regarding the removal of the same lesion, laparoscopic surgery is more efficient in reducing the inflammatory responses of the body and stabilize the inflammatory factors of cells (9). Sex hormones reflect the ovarian function to a certain extent (29). In this study, the ovarian function of patients was compared between the experimental and control group. The expression levels of FSH and LH were higher after surgery than those before surgery, while the expression level of $E_{2}$ was lower than that before surgery, which suggests that the ovarian function was restored to some extent. Patients in the experimental group had lower expression levels of FSH and LH than those in the control group, while higher expression level of $\mathrm{E}_{2}$ than those in the control group. This indicates that laparoscopic surgery has small trauma, and patients recover better, which is similar to the results of the study by Dong (11). However, it has also been reported that there is no significant difference in serum FSH, LH and $\mathrm{E}_{2}$ levels between patients undergoing laparoscopic and abdominal myomectomy (30), which is different from the results of the present study. The possible reason of this difference may be that both surgeries have good effects, however, there are differences in the description of ovarian function test results due to various factors, such as surgery and different time points to detect serum. However, the results of this study mainly show that myomectomy has a short-term effect on ovarian function, and patients generally recover in 6-12 months. In the present study, the incidence of postoperative complications of patients was compared between the two groups. Laparoscopic surgery can significantly reduce the incidence of postoperative complications, which is also conducive in improving the patients' postoperative quality of life as well as their prognosis. Liang et al (31) found that minimally invasive laparoscopic myomectomy has fewer postoperative complications than conventional abdominal myomectomy. Campo et al (32) reported that the pregnancy outcome of laparoscopic myomectomy for uterine fibroids was significantly better than abdominal myomectomy. In this study, 3 years after surgery, patients in the experimental group had higher pregnancy rate than those in the control group, indicating that laparoscopic surgery can improve the pregnancy rate and has important reference value for the selection of patients with uterine fibroid in childbearing age. The reason may be due to the small trauma of laparoscopic surgery, the small impact on the patient's abdominal environment, and the low incidence of postoperative complications. Patients with fertility requirements received infertility treatment. However, only the pregnancy rate was counted in this study, and the delivery was not taken into consideration, thus, further clinical observation is required.

In the present study, the effect of laparoscopic surgery on the serum levels of IL- 6 and TAC, and the ovarian function was comprehensively analyzed. Laparoscopic myomectomy has certain deficiencies. During surgery, it may be difficult to completely clean the fibroids, control the intraoperative bleeding and operate. Therefore, in practice, some patients due to undergo laparoscopic surgery finally undergo laparotomy (33). Also, in clinical applications, the surgery skills of medical personnel is important to improve the clinical effect of laparoscopic surgery.

In conclusion, reducing its effect on the ovarian function, serum pain index and oxidative damage index of patients with uterine fibroids, the laparoscopic myomectomy also reduces the incidence of postoperative complications and blood loss, and increases the pregnancy rate. Therefore, laparoscopic myomectomy has a high application value in clinical practice.

\section{Acknowledgements}

Not applicable.

\section{Funding}

No funding was received.

\section{Availability of data and materials}

The datasets used and/or analyzed during the present study are available from the corresponding author on reasonable request.

\section{Authors' contributions}

YaH and LY performed ELISA. FX and LX performed the laparoscopic myomectomy. FL and CC were responsible for the follow up. YaH and YuH assisted with statistical analysis. 
YaH wrote the manuscript. All authors read and approved the final manuscript.

\section{Ethics approval and consent to participate}

The study was approved by the Ethics Committee of the Central Hospital of Wuhan, Tongji Medical College, Huazhong University of Science and Technology (Wuhan, China). Patients who participated in this study had complete clinical data. Signed informed consents were obtained from the patients or their guardians.

\section{Patient consent for publication}

Not applicable.

\section{Competing interests}

The authors declare that they have no competing interests.

\section{References}

1. Thyagarajan MS and Sharif K: Space occupying lesions in liver. Indian J Pediatr 83: 1291-1302, 2016.

2. Saccardi C, Gizzo S, Noventa M, Ancona E, Borghero A and Litta PS: Limits and complications of laparoscopic myomectomy: Which are the best predictors? A large cohort single-center experience. Arch Gynecol Obstet 290: 951-956, 2014.

3. Evans P and Brunsell S: Uterine fibroid tumors: Diagnosis and treatment. Am Fam Physician 75: 1503-1508, 2007.

4. Sparić R: Uterine myomas in pregnancy, childbirth and puerperium. Srp Arh Celok Lek 142: 118-124, 2014 (In Serbian).

5. Ciavattini A, Di Giuseppe J, Stortoni P, Montik N, Giannubilo SR, Litta P, Islam MS, Tranquilli AL, Reis FM and Ciarmela P: Uterine fibroids: Pathogenesis and interactions with endometrium and endomyometrial junction. Obstet Gynecol Int 2013: 173184, 2013.

6. Florio P, Filippeschi M, Imperatore A, Mereu L, Franchini M, Calzolari S, Mencaglia L and Litta P: The practicability and surgeons' subjective experiences with vaginal danazol before an operative hysteroscopy. Steroids 77: 528-533, 2012.

7. Arthur R, Kachura J, Liu G, Chan C and Shapiro H: Laparoscopic myomectomy versus uterine artery embolization: Long-term impact on markers of ovarian reserve. J Obstet Gynaecol Can 36: 240-247, 2014

8. Han CM, Lee CL, Su H, Wu PJ, Wang CJ and Yen CF: Single-port laparoscopic myomectomy: Initial operative experience and comparative outcome. Arch Gynecol Obstet 287: 295-300, 2013.

9. Li MH, Leng JH, Shi JH, Jia SZ and Lang JH: Comparison of postoperative residue, recurrence and pregnancy outcome between laparoscopic and transabdominal myomectomy. Zhonghua Fu Chan Ke Za Zhi 46: 669-673, 2011 (In Chinese).

10. Dubuisson JB, Fauconnier A, Deffarges JV, Norgaard C, Kreiker G and Chapron C: Pregnancy outcome and deliveries following laparoscopic myomectomy. Hum Reprod 15: 869-873, 2000.

11. Dong J: Influence of laparoscopic myomectomy on ovarian function and serum trauma related indexes. J Hainan Med Univ 20: 1103-1104, 2014 (In Chinese).

12. Chen J, Chen W, Zhang L, Li K, Peng S, He M and Hu L: Safety of ultrasound-guided ultrasound ablation for uterine fibroids and adenomyosis: A review of 9988 cases. Ultrason Sonochem 27: 671-676, 2015.

13. Cela V, Freschi L, Simi G, Tana R, Russo N, Artini PG and Pluchino N: Fertility and endocrine outcome after robot-assisted laparoscopic myomectomy (RALM). Gynecol Endocrinol 29: 79-82, 2013.

14. Wang Q and Luo J: Effect of laparoscopic myomectomy on inflammatory reaction,immune function, stress hormones and ovarian function. J Hainan Med Univ 24: 37-40, 2018 (In Chinese).

15. Yuan G, Zeng CL, Zhu DD and Shi XJ: Influences of RFA combined with TACE on the HIF-1 $\alpha$ and EGR level of patients with primary hepatic carcinoma. Eur Rev Med Pharmacol Sci 21: 1738-1745, 2017.
16. Taniguchi F, Koike N, Kikukawa T, Yabuta M, Yamaguchi M, Adachi E and Nakayama T: An evaluation of laparoscopic hysterectomy alone versus in combination with laparoscopic myomectomy for patients with uterine fibroids. Eur J Obstet Gynecol Reprod Biol 210: 132-138, 2017.

17. Radosa JC, Radosa CG, Mavrova R, Wagenpfeil S, Hamza A, Joukhadar R, Baum S, Karsten M, Juhasz-Boess I, Solomayer EF, et al: Postoperative quality of life and sexual function in premenopausal women undergoing laparoscopic myomectomy for symptomatic fibroids: A prospective observational cohort study. PLoS One 11: e0166659, 2016.

18. Aktas GE, Sarıkaya A, Can N and Demir SS: Microcalcified hepatic metastases incidentally detected on F-18 NaF PET/CT in a patient with prostate cancer. Nucl Med Mol Imaging 51: 364-367, 2017.

19. Abdusattarova K, Mettler L, Alkatout I and Dempfle A: Endoscopic treatment of symptomatic fibroids at reproductive age and beyond. Minim Invasive Ther Allied Technol 26: 355-361, 2017.

20. Bean EM, Cutner A, Holland T, Vashisht A, Jurkovic D and Saridogan E: Laparoscopic myomectomy: A single-center retrospective review of 514 patients. J Minim Invasive Gynecol 24: 485-493, 2017

21. Tatsuaki S, Yasuo S, Takehiro O, Yasuhiro H, Yoshihiro N, Michihiko K, Kenta S, Yuji N and Taijiro S: Multidetector CT in detection of troublesome posterior sectoral hepatic duct communicating with cystic duct. Br J Radiol 90: 20170260, 2017.

22. Yamaguchi K, Kumakura S, Murakami T, Someya A, Inada E and Nagaoka I: Ketamine suppresses the substance P-induced production of IL- 6 and IL- 8 by human U373MG glioblastoma/astrocytoma cells. Int J Mol Med 39: 687-692, 2017.

23. Kim SY, Koo BN, Shin CS, Ban M, Han K and Kim MD: The effects of single-dose dexamethasone on inflammatory response and pain after uterine artery embolisation for symptomatic fibroids or adenomyosis: A randomised controlled study. BJOG 123: 580-587, 2016

24. Cojocaru IM, Cojocaru M, Sapira V and Ionescu A: Evaluation of oxidative stress in patients with acute ischemic stroke. Rom J Intern Med 51: 97-106, 2013.

25. Jiang X, Zhong P, Huang C, Meng Y, He F and Fan X: Expression and clinical significance of oxidative stress markers in serum of patients with stable chronic obstructive pulmonary disease. Zhongguo Hu Xi Yu Wei Zhong Jian Hu Za Zhi 15: 542-547, 2016 (In Chinese).

26. Hip Fracture Accelerated Surgical Treatment and Care Track (HIP ATTACK) Investigators: Accelerated care versus standard care among patients with hip fracture: The HIP ATTACK pilot trial. CMAJ 186: E52-E60, 2014.

27. Hahn M, Brucker S, Kraemer D, Wallwiener M, Taran FA, Wallwiener CW and Krämer B: Radiofrequency volumetric thermal ablation of fibroids and laparoscopic myomectomy: Long-term follow-up from a randomized trial. Geburtshilfe Frauenheilkd 75: 442-449, 2015.

28. Brucker SY, Hahn M, Kraemer D, Taran FA, Isaacson KB and Krämer B: Laparoscopic radiofrequency volumetric thermal ablation of fibroids versus laparoscopic myomectomy. Int $\mathrm{J}$ Gynaecol Obstet 125: 261-265, 2014

29. Wang Y, Ji W, Zhang X and Tan J: Laparoscopic liver resection and enucleation of liver hemangioma with selective hepatic vascular occlusion: Technique and indications. J Laparoendosc Adv Surg Tech A 27: 944-950, 2017.

30. Song Y, Wang Y and Zhang X: Clinical effectiveness and safety of laparoscopic myomectomy in patients with fibroid. Chin Mod Doc 56: 52-54, 2018 (In Chinese).

31. Liang YZ, Du Z and Chen ZM: Comparison of curative effect of minimally invasive laparoscopic uterine myoma with conventional open surgery for treatment of uterine fibroids. Chin J Clin Oncol Rehabil 23: 209-211, 2016 (In Chinese).

32. Campo S, Campo V and Gambadauro P: Reproductive outcome before and after laparoscopic or abdominal myomectomy for subserous or intramural myomas. Eur J Obstet Gynecol Reprod Biol 110: 215-219, 2003.

33. Yuan H, Wang C, Wang D and Wang Y: Comparing the effect of laparoscopic supracervical and total hysterectomy for uterine fibroids on ovarian reserve by assessing serum anti-mullerian hormone levels: A prospective cohort study. J Minim Invasive Gynecol 22: 637-641, 2015.

This work is licensed under a Creative Commons Attribution-NonCommercial-NoDerivatives 4.0 International (CC BY-NC-ND 4.0) License. 